\title{
The impact of diabetes on coronary heart disease differs from that on ischaemic stroke with regard to the gender
} Marjukka Hyvärinen*1, Jaakko Tuomilehto ${ }^{1,2}$, Tiina Laatikainen², Stefan Söderberg ${ }^{3}$, Mats Eliasson ${ }^{3}$, Peter Nilsson ${ }^{4}$ and Qing Qiao ${ }^{1,2}$

\author{
Address: ${ }^{1}$ Department of Public Health, University of Helsinki, Helsinki, Finland, ${ }^{2}$ Diabetes Prevention Unit, Department of Chronic Disease \\ Prevention, National Institute for Health and Welfare, Helsinki, Finland, ${ }^{3}$ Department of Public Health and Clinical Medicine, Umeå University \\ Hospital SE-901 85 Umeå, Sweden and ${ }^{4}$ Department of Clinical Sciences, Lund University University Hospital, S-205 02 Malmö, Sweden \\ Email: Marjukka Hyvärinen* - marjukka.hyvarinen@helsinki.fi; Jaakko Tuomilehto - jaakko.tuomilehto@ktl.fi; \\ Tiina Laatikainen - tiina.laatikainen@ktl.fi; Stefan Söderberg - stefan.soderberg@medicin.umu.se; Mats Eliasson - mats.eliasson@nll.se; \\ Peter Nilsson - peter.nilsson@med.lu.se; Qing Qiao - qing.qiao@ktl.fi \\ * Corresponding author
}

Published: 24 March 2009

Cardiovascular Diabetology 2009, 8:17 doi:10.1186/1475-2840-8-17

This article is available from: http://www.cardiab.com/content/8/I/I7

(c) 2009 Hyvärinen et al; licensee BioMed Central Ltd.

This is an Open Access article distributed under the terms of the Creative Commons Attribution License (http://creativecommons.org/licenses/by/2.0), which permits unrestricted use, distribution, and reproduction in any medium, provided the original work is properly cited.
Received: 18 February 2009

Accepted: 24 March 2009

\begin{abstract}
Background: To study the diabetes related CVD risk between men and women of different ages.

Methods: Hazards ratios (HRs) $(95 \% \mathrm{Cl})$ for acute $\mathrm{CHD}$ and ischaemic stroke events were estimated based on data of Finnish and Swedish cohorts of 5 III women and 4167 men.

Results: I 82 (3.6\%) women and 348 (8.4\%) men had CHD and I 29 (2.5\%) women and I 37 (3.3\%) men ischaemic stroke events. The multivariate adjusted HRs for acute CHD at age groups of $40-$ $49,50-59$ and 60-69 years were 1.00 (1.94), I.78 (4.23), 3.75 (8.40) in women (men) without diabetes and 4.35 (5.40), 5.49 (9.54) and 8.84 (13.76) in women (men) with diabetes. The corresponding HRs for ischaemic stroke were I.00 (I.26), 2.48 (2.83) and 5.17 (5.II) in women (men) without diabetes and 4.14 (4.9I), 3.32 (6.75) and 13.9I (I8.06) in women (men) with diabetes, respectively.
\end{abstract}

Conclusion: CHD risk was higher in men than in women but difference reduced in diabetic population. Diabetes, however, increased stroke risk more in men than in women.

\section{Background}

Coronary heart disease (CHD) and stroke are common causes of disability and death [1]. Diabetes mellitus has been found to increase the risk of CHD and stroke events both in women and in men [2-8]. A more markedly increased relative risk for CHD $[2,4,5]$ and stroke [6-8] has been found in diabetic women compared with diabetic men, however the reported magnitudes vary greatly between studies $[2,7,8]$.
CHD and stroke are generally discussed as two diseases with similar etiology caused by atherosclerotic vascular disease. While CHD and stroke have been shown to share some known cardiovascular disease (CVD) risk factors [1], some common CHD risk factors, such as serum total cholesterol $[9,10]$ levels have not been indicated to have an association with increased risk of stroke [10,11]. Also, MONICA study reported CHD rates to decrease and stroke rates to increase in some populations while in other pop- 
ulations the opposite was observed [12]. This may imply different underlying mechanisms and etiology for the two diseases. To what extent diabetes, gender and age affect the development of CHD and stroke is not fully elucidated. Furthermore, little is known about the magnitude of the association between CVD risk factors and the risk of CHD and ischaemic stroke.

The purpose of the present study was to estimate the impact of diabetes and gender and age on the development of CHD and ischaemic stroke and to find out to what extent the known CVD risk factors affect the development of these diseases.

\section{Participants and methods}

This sub-data analysis of the DECODE study comprised of 9278 participants, 5111 (55.1\%) women and 4167 (44.9\%) men, from 7 cohorts from Finland and Sweden. Individuals participating at the baseline survey were followed up till 2006 and 2004 for the Finnish and the Swedish cohorts respectively. The age range of the study population varied from 40 to 69 years. Measurements for fasting plasma glucose and $75 \mathrm{~g} 2$-h oral glucose tolerance test, body mass index (BMI), total serum cholesterol, HDL cholesterol, systolic and diastolic blood pressure, antihypertensive treatment, and smoking status were available for each study population included in the current analysis.

\section{Definition of the clinical end points}

Acute CHD and ischaemic stroke events (both fatal and non-fatal) were used as end points. Individuals with a history of CVD before the baseline survey were excluded from the analysis. The information on CVD mortality and morbidity were collected from National Causes of Death Register and the National Hospital Discharge Registry and ascertained by using a computerized record linkage of individual ID numbers of each of the individuals participating in the study. The acute CHD and ischaemic stroke events were coded according to the International Classification of Diseases $\left(8^{\text {th }}, 9^{\text {th }}\right.$ revisions and $10^{\text {th }}$ revision) with codes 410 to 414 and I20 to I25 for fatal and codes 410 to 411 and I21 to I22 for non-fatal CHD and codes 433, 434 and I63 for ischaemic stroke. Cases of haemorrhagic stroke were checked and not included in the final data analysis due to the small numbers. Diabetes was defined as either a history of diabetes at baseline or FPG levels $>=7.0 \mathrm{mmol} / \mathrm{L}$ and $/$ or 2-h PG levels $>=11.1 \mathrm{mmol} /$ L. Participants who had emigrated and whose vital status could not be confirmed were treated as censored cases.

The local Ethics Committees had approved each individual study plan and the data analysis plan was approved by the Ethics Committee of the National Public Health Institute, Finland.

\section{Statistical methods}

The data analysis was carried out by using SPSS for Windows version 15.0. Event rates per 1000 person-years for CHD and ischaemic stroke were calculated for per 10-year age-groups (40-69 years), gender and diabetic status. Differences between women and men within each of the age categories were estimated using the $\chi^{2}$ test. Univariate analysis of variance was used to estimate the trend in cardiovascular risk factors with aging. Hazard ratios with 95\% confidence intervals for acute CHD or ischaemic stroke events were estimated using Cox proportional hazards model. The analysis was adjusted for study, hypertension status ( $\geq 140 / 90 \mathrm{mmHg}$ or treatment), body mass index (BMI), total serum cholesterol, HDL-cholesterol and smoking status. BMI was calculated by using weight in kilograms divided by the square of height in meters and smoking status was categorized as current smoker, exsmoker or nonsmoker. Non-diabetic women aged 40-49 years were used as the reference group for risk calculations.

\section{Results}

The demographic data at baseline and the follow-up information in each cohort are shown in table 1 . The maximum length of follow-up varied between 4.9 to 20.6 years in women and in men. During this period 182 (3.6\%) women and $348(8.4 \%)$ men had acute CHD and $129(2.5 \%)$ women and 137 (3.3\%) men acute ischaemic stroke events. A total of $384(7.5 \%)$ women and 442 $(10.6 \%)$ men had diabetes (both diagnosed and undiagnosed) (table 1). The characteristics of subjects according to age groups, diabetes status and gender are given in table 2 . In the non-diabetic population, the means of BMI and total cholesterol as well as the prevalence of hypertension were all higher in men than in women in the youngest age group, but the gender differences leveled off with increasing age and became even or lower in men than in women in the oldest age group. In non-diabetic women and men the increasing trend with aging was significant $(\mathrm{p}=0.001)$ for BMI, cholesterol and hypertension (table 2). Diabetic women were, however, more obese than diabetic men in all ages. HDL-cholesterol was higher in women than in men and smoking was more common in men than in women in all ages regardless of the diabetic status. The increasing trend in known CVD risk factors with aging in diabetic individuals was significant only for total cholesterol levels in women.

The event rates for acute CHD and ischaemic stroke were higher in men with and without diabetes compared with their female counterparts. The gender difference for CHD was larger in the non-diabetic than in the diabetic individuals, whereas this was not as substantial for ischaemic stroke as for CHD (table 3). The multivariate-adjusted hazard ratios of acute CHD events increased with aging 
and diabetes, and were higher in men both with and without diabetes compared with age matched women, but the gender difference was diminished in diabetic individuals. In contrast, the gender difference with the ischaemic stroke was enlarged in the diabetic population because the risk of ischaemic stroke increased more in diabetic men than in diabetic women (table 3). Diabetes and aging were associated with an increased risk of acute CHD and ischaemic stroke events in both genders, however diabetes was a stronger risk predictor for the risk of ischaemic stroke than for CHD (table 4). Cholesterol predicted the risk of acute CHD, but not ischaemic stroke event, in both genders. Hypertension increased the acute CHD and ischaemic stroke risk, and BMI CHD risk only in women (table 4).

\section{Discussion}

In the present study we found that both non-diabetic and diabetic men had a higher risk of CHD than their female counterparts, but the gender difference was smaller in the diabetic than in the non-diabetic populations. In contrast, diabetic men had higher rates of ischaemic stroke events than diabetic women but the event rates did not differ much in the non-diabetic population. Diabetes conveyed a higher CHD risk in women but ischaemic stroke risk in men.

Diabetes is a well known risk factor for CHD [13-15] and stroke $[16,7,8]$ in both genders. CHD and stroke are often discussed as diseases of the same family caused by atherosclerotic vascular disease. While this would implicate shared risk factors, studies have indicated some differences in risk factor profiles for the two diseases [9-11]. Also, the patterns of the trend of CHD and stroke incidence have been shown to vary in a population $[12,17]$. In the present study the diabetes attributable risk was higher to the acute ischaemic stroke than to the acute CHD in men, whereas it was similar in women. Previous studies have reported diabetes to increase the risk of CHD $[2,4,5]$ and stroke $[6-8]$ more markedly in women than in men. These studies have, however, used non-diabetic women and non-diabetic men as reference groups for women and for men, respectively, when they calculated the relative risk for diabetic individuals. Since the nondiabetic young men had much higher CHD and stroke risk than non-diabetic women, the relative risk increase due to diabetes in men tended to be lower than that in women. To make it comparable between women and men, we have used non-diabetic women in the youngest age group as reference for both women and men of all other age groups. We found the relative risk was higher in men than in women both with and without diabetes, even though the gender difference for the CHD risk was diminished in diabetic individuals. Contradictory to the previous reports on stroke we found in the present study a more markedly increased risk of acute ischaemic stroke events in diabetic men compared with diabetic women. But whether this is true for haemorrhagic stroke or for the subtypes of ischaemic stroke cannot be examined in the current study due to the low number of the haemorrhagic stroke events and inability of further classification of individuals into subtypes of the ischaemic stroke. Previous studies with both ischaemic and haemorrhagic stroke showed a higher relative stroke risk in diabetic women than in diabetic men [6-8], and it was also reported that the risk for certain subtypes of ischaemic or haemorrhagic stroke differed between women and men $[18,19]$. This might have affected the observations and needs to be explored in more detail in the future.

Non-diabetic men had higher rates of smoking, hypertension and abnormalities of lipid profiles than non-diabetic women; which could partly explain the higher CHD risk in non-diabetic men than in non-diabetic women. Also, other factors such as the menopause status [20] and the management of risk factors $[20,21]$ may have contributed to the observed gender difference in CHD and ischemic stroke incidence. Obesity and cholesterol levels were higher in diabetic women than diabetic men, which may partly have reduced the gender difference in CHD risk in diabetic population. Aging seemed a strong risk predictor for CHD in both women and men, particularly for men. This may to some extent be explained by the fact that men are exposed to the CVD risk factors early in the life than women. Even though CHD and stroke have some common aspects, the reactivity of coronary and cerebral arteries to the CVD risk factors, environmental and genetic factors, has been found to differ [22]. Thus, as found in the present study the known CVD risk factors including diabetes may have contributed differently to the CHD and the ischaemic stroke, and partly explained the gender difference in the risk of CHD and ischaemic stroke in diabetic individuals. However, when we interpret the results we need to bear in mind that the number of ischaemic stroke events in the present study was low, particularly in the younger age groups both in women and in men.

The strengths of the present study are the long length of follow-up and the pooled data from different cohorts, which gave a large sample size and increased statistical power. In order to make up for the differences in between the different study centers all the analysis were adjusted for "cohort". Even though the sample size was relatively large, the follow-up length for many of the cohorts is still short with low numbers of the events accumulated.

\section{Conclusion}

In summary, the CHD and ischaemic stroke risk was higher in men than in women with and without diabetes, however, the gender difference diminished for CHD but 
enlarged for ischaemic stroke in diabetic individuals. The known risk factors including diabetes have contributed differently to the development of CHD and ischaemic stroke in women and men. The finding may help to make strategies to manage diabetes, CHD and stroke in women and in men.

\section{Abbreviations}

BMI: body mass index; CHD: coronary heart disease; CVD: cardiovascular disease; DECODE: Diabetes Epidemiology: Collaborative analysis Of Diagnostic criteria in Europe; FPG: fasting plasma glucose; 2-h PG: plasma glucose 2 -h after 75-g glucose load

\section{Competing interests}

The authors declare that they have no competing interests.

\section{Authors' contributions}

$\mathrm{MH}$ ran the data analysis and drafted the manuscript. QQ participated in the concept, funding, design and coordination of the study and in drafting of the manuscript. TL, SS and ME took part in the data collection, drafted and approved the final version of the manuscript. PN drafted and approved the final version of the manuscript. JT was responsible for the DECODE collaboration, funding and the conception of the manuscript and approved the final version of the manuscript.

\section{Additional material}

\section{Additional file 1}

Demographic and follow-up information of the study populations included.

Click here for file

[http://www.biomedcentral.com/content/supplementary/14752840-8-17-S1.doc]

\section{Additional file 2}

Baseline characteristics and the incidence of cardiovascular end points in individuals according to age groups, gender, and diabetic status. Click here for file

[http://www.biomedcentral.com/content/supplementary/1475-

2840-8-17-S2.doc]

\section{Additional file 3}

Event rates per 1000 person-years and hazard ratios (95\% confidence intervals) for acute CHD and ischemic stroke events by diabetic status.

Click here for file

[http://www.biomedcentral.com/content/supplementary/14752840-8-17-S3.doc]

\section{Additional file 4}

Hazard ratios (95\% confidence intervals) corresponding to a one SD increase in continuous variables or as indicated.

Click here for file

[http://www.biomedcentral.com/content/supplementary/14752840-8-17-S4.doc]

\section{Acknowledgements}

We would like to thank Professor Kalevi Pyörälä for useful advice in the planning and preparation of the manuscript. This analysis has been carried out with the help of grants from Finnish Academy ( I I8492). There were no conflicts of interest.

\section{Organisation}

The DECODE Study (Diabetes Epidemiology: Collaborative analysis Of Diagnostic Criteria in Europe) was started in 1997 upon the initiative of the European Diabetes Epidemiology Group.

\section{Studies and investigators in this collaborative study are:}

Finland The National FINRISK 87 and 92 cohorts: J. Tuomilehto 1,2,3, P. Jousilahti ${ }^{2}$, J. Lindström²,. I. Department of Public Health, University of Helsinki, Helsinki; 2. Department of Health Promotion and Chronic Disease Prevention, National Public Health Institute, Helsinki; 3. South Ostrobothnia Central Hospital, Seinäjoki.

The National FINRISK 2002: J. Tuomilehto, T. Laatikainen, M. Peltonen and J. Lindström, Department of Health Promotion and Chronic Disease Prevention, National Public Health Institute, Helsinki

Vantaa Study: R. Tilvis', S. Sairanen', J. Tuomilehto $2,3,4$, I. Division of geriatrics, Department of Medicine, University of Helsinki, Helsinki. 2. Department of Health Promotion and Chronic Disease Prevention, National Public Health Institute, Helsinki; 3. Department of Public Health, University of Helsinki, Helsinki; 4. South Ostrobothnia Central Hospital, Seinäjoki.

Sweden Northern Swedish MONICA: M. Eliasson, B. Stegmayr, S. Söderberg. Department of Public Health and Clinical Medicine, Umeå University, Umeå.

\section{Secretariat:}

Q. Qiao', K. Borch-Johnsen², J. Tuomilehto', I. Diabetes Unit, Department of Health Promotion and Chronic Disease Prevention, National Public Health Institute, Helsinki and Department of Public Health, University of Helsinki, Helsinki, Finland. 2. Steno Diabetes Center, Gentofte, Denmark.

Data analysis:

M. Hyvärinen', Q. Qiao',2 I. Department of Public Health, University of Helsinki, Helsinki, Finland; 2. Diabetes Unit, Department of Health Promotion and Chronic Disease Prevention, National Public Health Institute, Helsinki, Finland.

\section{References}

I. Rosamond W, Flegal K, Friday G, Furie K, Go A, Greenlund K, Haase N, Ho M, Howard V, Kissela B, Kittner S, Lloyd-Jones D, McDermott M, Meigs J, Moy C, Nichol G, O'Donnell C], Roger V, Rumsfeld J, Sorlie P, Steinberger J, Thom T, Wasserthiel-Smoller S, Hong Y, Ameri- 
can Heart Association Statistics Committee and Stroke Statistics Subcommittee: Heart disease and stroke statistics - 2008 update: a report from the American Heart Association Statistics Committee and Stroke Statistics Subcommittee. Circulation 2008, I I 7:e25- | 46.

2. Lee WL, Cheung AM, Cape D, Zinman B: Impact of diabetes on coronary artery disease in women and men: a meta-analysis of prospective studies. Diabetes Care 2000, 23:962-968.

3. Kanaya AM, Grady D, Barrett-Connor E: Explaining the sex difference in coronary heart disease mortality among patients with type 2 diabetes mellitus: a meta-analysis. Arch Intern Med 2002, 162:1737-1745.

4. Yusuf S, Hawken S, Ounpuu S, Dans T, Avezum A, Lanas F, McQueen M, Budaj A, Pais P, Varigos J, Lisheng L: INTERHEART Study Investigators. Effect of potentially modifiable risk factors associated with myocardial infarction in 52 countries (the INTERHEART study): case-control study. Lancet 2004, 364:937-952.

5. Huxley R, Barzi F, Woodward M: Excess risk of fatal coronary heart disease associated with diabetes in men and women: meta-analysis of $\mathbf{3 7}$ prospective cohort studies. BMJ 2006, 332:73-78.

6. Lehto S, Rönnemaa T, Pyörälä K, Laakso M: Predictors of stroke in middle-aged patients with non-insulin-dependent diabetes. Stroke 1996, 27:63-68.

7. Hart CL, Hole DJ, Smith GD: Comparison of risk factors for stroke incidence and stroke mortality in $\mathbf{2 0}$ years of followup in men and women in the Renfrew/Paisley Study in Scotland. Stroke 2000, 3 I: 1893-1896.

8. Almdal T, Scharling H, Jensen JS, Vestergaard $H$ : The independent effect of type 2 diabetes mellitus on ischemic heart disease, stroke, and death: a population-based study of 13,000 men and women with 20 years of follow-up. Arch Intern Med 2004, 164: $1422-1426$

9. Wilson PW, D'Agostino RB, Levy D, Belanger AM, Silbershatz $H$, Kannel WB: Prediction of coronary heart disease using risk factor categories. Circulation 1998, 97:1837-1847.

10. Wilhelmsen L, Köster M, Harmsen P, Lappas G: Differences between coronary disease and stroke in incidence, case fatality, and risk factors, but few differences in risk factors for fatal and non-fatal events. Eur Heart J 2005, 26:1916-1922.

II. Bots ML, Elwood PC, Nikitin Y, Salonen JT, Freire de Concalves A, Inzitari D, Sivenius J, Benetou V, Tuomilehto J, Koudstaal PJ, Grobbee DE: Total and HDL cholesterol and risk of stroke. EUROSTROKE: a collaborative study among research centres in Europe. J Epidemiol Community Health 2002, 56(SuppI I):i I 9-24.

12. Truelsen T, Mähönen M, Tolonen H, Asplund K, Bonita R, Vanuzzo D: WHO MONICA Project. Trends in stroke and coronary heart disease in the WHO MONICA Project. Stroke 2003, 34: $1346-1352$.

13. Jousilahti P, Vartiainen E, Tuomilehto J, Puska P: Sex, Age, Cardiovascular Risk Factors, and Coronary Heart Disease: A Prospective Follow-Up Study of I4 786 Middle-Aged Men and Women in Finland. Circulation 1999, 99: I 165-1 I72

14. Laakso M: Cardiovascular disease in type 2 diabetes: challenge for treatment and prevention. J Intern Med 200I, 249:225-235.

15. Howard BV, Best LG, Galloway JM, Howard WJ, Jones K, Lee ET, Ratner RE, Resnick HE, Devereux RB: Coronary heart disease risk equivalence in diabetes depends on concomitant risk factors. Diabetes Care 2006, 29:391-397.

16. Tuomilehto J, Rastenyte D, Jousilahti P, Sarti C, Vartiainen E: Diabetes Mellitus as a Risk Factor for Death From Stroke. Prospective Study of the Middle-aged Finnish Population. Stroke 1996, 27:210-215.

17. Kitamura A, Iso H, lida M, Naito $Y$, Sato S, Jacobs DR, Nakamura M, Shimamoto $T$, Komachi $Y$ : Trends in the incidence of coronary heart disease and stroke and the prevalence of cardiovascular risk factors among Japanese men from I963 to I994. Am J Med 2002, I I 2:104-109.

18. Roquer J, Campello AR, Gomis M: Sex Differences in first-ever acute stroke. Stroke 2003, 34: I58I-I585.

19. Thrift AG, Dewey HM, Sturm JW, Srikanth VK, Gilligan AK, Gall SL, Macdonell RA, McNeil JJ, Donnan GA: Incidence of Stroke Subtypes in the North East Melbourne Stroke Incidence Study (NEMESIS): Differences between Men and Women. Neuroepidemiology 2009, 32: I I-I8.
20. Andreotti F, Marchese N: Women and coronary disease. Heart 2008, 94:108-II6.

21. Jairath $\mathrm{N}$ : Implications of gender differences on coronary artery disease risk reduction in women. AACN Clin Issues 200I, 1 2: 17-28.

22. Puddu P, Puddu GM, Bastagli L, Massarelli G, Muscari A: Coronary and cerebrovascular atherosclerosis: two aspects of the same disease or two different pathologies? Arch Gerontol Geriatr 1995, 20:15-22.
Publish with Bio Med Central and every scientist can read your work free of charge

"BioMed Central will be the most significant development for disseminating the results of biomedical research in our lifetime. "

Sir Paul Nurse, Cancer Research UK

Your research papers will be:

- available free of charge to the entire biomedical community

- peer reviewed and published immediately upon acceptance

- cited in PubMed and archived on PubMed Central

- yours - you keep the copyright

Submit your manuscript here:

http://www.biomedcentral.com/info/publishing_adv.asp
BiolMedcentral 